\title{
Cabazitaxel in the treatment of metastatic castration-resistant prostate cancer: patient selection and special considerations
}

This article was published in the following Dove Press journal:

OncoTargets and Therapy

17 August 2017

Number of times this article has been viewed

\author{
Sheel A Patel \\ Jean Hoffman-Censits \\ Department of Medical Oncology, \\ Sidney Kimmel Cancer Center, \\ Thomas Jefferson University, \\ Philadelphia, PA, USA
}

\begin{abstract}
Cabazitaxel is an effective chemotherapeutic agent used in the treatment of metastatic castration-resistant prostate cancer (mCRPC) refractory to docetaxel. With the advent of new antiandrogen therapies, immune-based treatments, and radioactive-targeted therapy, there are now multiple effective and approved agents for this disease state. The optimal sequencing of these agents is unclear as there are no large-scale head-to-head comparisons. Clinicians must familiarize themselves with the most recent studies as well as drug toxicities to determine the best treatment option for their patients. In this review, we focus on the development of cabazitaxel for mCRPC, evaluate its efficacy, and highlight key strategies for toxicity management. Additionally, we summarize the studies that address cabazitaxel treatment sequencing and optimal dosing schedule.
\end{abstract}

Keywords: sequencing, clinical trials, docetaxel, abiraterone, enzalutamide, biomarkers

\section{Introduction}

Prostate cancer is the second most common tumor in men in the United States. The annual mortality rate has slowly declined over the past years possibly related to improved screening and curative treatment for early stage disease. Unfortunately, men with metastatic disease have a 5 -year survival rate of only $28 \%$ compared to $\sim 100 \%$ for local and regional stage diseases. ${ }^{1}$ This large discrepancy is due to the development of castrate-resistant metastatic disease, a more aggressive lethal phenotype with a distinct biology from castrate-sensitive tumors.

Until this past decade, there were few treatment options available to clinicians treating metastatic castration-resistant prostate cancer (mCRPC). The leading treatments were estramustine and mitoxantrone, both of which had little objective data for response. In 2004, the US Food and Drug Administration (FDA)-approved docetaxel as the first chemotherapeutic agent with overall survival (OS) benefit in mCRPC. ${ }^{2}$ In the past 6 years, the treatment arsenal of agents that improve OS in MCRPC has rapidly expanded to include sipuleucel-T, abiraterone, enzalutaminde, radium-223, and cabazitaxel. Currently, these agents are given sequentially, at the discretion of the treating physician, with little objective information other than the medical comorbidities and choice of the patient, in driving treatment sequence decisions. Although in the future, biomarker data may more globally inform sequencing of prostate cancer therapy, currently physician and patient preference drive most treatment decisions.

In this review, we focus on the clinical development of cabazitaxel and analyze data demonstrating its benefit on OS in men with mCRPC and data that may help
Correspondence: Jean Hoffman-Censits Department of Medical Oncology, Sidney Kimmel Cancer Center, Thomas Jefferson University, Jefferson Medical College Building, Suite 700, 1025 Walnut Street, Philadelphia, PA 19107, USA

Tel +I 2I5 $50364 \mid 3$

$\mathrm{Fax}+\mathrm{I} 2155033408$

Email jean.hoffman-censits@jefferson.edu 
practitioners with sequencing, dose modifications, and patient selection for cabazitaxel therapy.

\section{Mechanism of action}

Taxane chemotherapy significantly changed the treatment landscape in mCRPC. In addition to inducing apoptosis by microtubule disruption, taxanes have been shown to decrease translocation of the androgen receptor (AR) to the nucleus in prostate cancer cells as well as decrease transcription of the AR protein. ${ }^{3}$ Docetaxel proved to be a potent semisynthetic taxane analog in $\mathrm{mCRPC}$, improving OS when compared to the historic standard mitoxantrone, and it remained the only standard in mCRPC for over a decade. ${ }^{2}$ Unfortunately, use of the drug was limited for many patients due to treatment intolerance from neuropathy, serositis, or refractory cytopenias. Additionally, acquired tumor resistance to docetaxel and cases of primary resistance were recognized as limitations for its continued use.

Cabazitaxel was developed due to its ability to overcome tumor resistance to taxanes. Preclinical models of multidrugresistant human and murine cancer cell lines demonstrated improved cytotoxicity with cabazitaxel compared to docetaxel. Unlike its parent drugs, cabazitaxel has poor binding to the adenosine triphosphate-dependent drug efflux pump P glycoprotein (P-gp) $1 .{ }^{4}$ This allows the drug to accumulate intracellularly at greater concentrations than docetaxel and is thought to be part of the mechanism for improved cabazitaxel cytoxicity. ${ }^{5}$

\section{Cabazitaxel clinical development}

Cabazitaxel (formerly XRP6258) was initially tested in a Phase I trial in 25 patients with metastatic solid malignancies, eight of whom had mCRPC. Participants had two or fewer previous lines of therapy and $32 \%$ had previous taxane exposure. ${ }^{4}$ Doses were escalated from $10 \mathrm{mg} / \mathrm{m}^{2}$ to the maximum tolerated dose of $25 \mathrm{mg} / \mathrm{m}^{2}$ and limited due to neutropenia events. Interestingly, four patients achieved a partial response, two of whom had mCRPC.

\section{Cabazitaxel in the second line and beyond}

These data supported the initiation of the international Phase III TROPIC trial, which opened in $2007 .{ }^{6}$ Seven hundred fifty-five men with mCRPC who had disease progression by Response Evaluation Criteria in Solid Tumor (RECIST) or prostate specific antigen (PSA) criteria during or after docetaxel were randomized to treatment with mitoxantrone $\left(12 \mathrm{mg} / \mathrm{m}^{2}\right)$ and prednisone $10 \mathrm{mg}$ daily vs cabazitaxel $\left(25 \mathrm{mg} / \mathrm{m}^{2}\right)$ and prednisone. Therapy was given once every 3 weeks, and the primary end point was OS.
The patient characteristics between both arms were comparable, with $50 \%$ of the study population having measurable soft tissue disease and $25 \%$ with visceral disease. Following enrollment of the first 59 patients, inclusion criteria were amended to exclude patients treated with $<225 \mathrm{mg} / \mathrm{m}^{2}$ cumulative dose of docetaxel, in accordance with updated docetaxel guidelines recommending at least 12 weeks of therapy prior to making treatment change decisions. With a median follow-up of 12.8 months, the primary end point of OS in the intent to treat population was 15.1 months in the cabazitaxel group compared to 12.7 months in the mitoxantrone group, demonstrating a 30\% relative risk reduction of death $(P<0.0001)$. Progression-free survival (PFS) was also statistically better in the cabazitaxel group compared with the mitoxantrone group (2.8 vs 1.4 months, $P<0.0001)$. A subgroup analysis showed that patients with measurable disease had significantly better tumor response rate $(14.1 \%$ vs $4.4 \%, P=0.0005)$ and PSA response rate with cabazitaxel compared with mitoxantrone $(39.2 \%$ vs $17.8 \%, P=0.0002)$. As demonstrated in the Phase I trial, there was a high rate of febrile neutropenia compared to mitoxantrone ( $8 \%$ vs $1 \%$ ). Nonhematological adverse events with cabazitaxel were similar to that in the Phase I study with $47 \%$ diarrhea and $37 \%$ fatigue. The rate of neuropathy in this cohort was only $14 \%$. In general, dose reductions were more common in the cabazitaxel group; however, more cycles of cabazitaxel were delivered compared to mitoxantrone (6 vs 4).

Though OS was improved with cabazitaxel, there were 18 treatment-related deaths compared to 9 deaths with mitoxantrone. The most common cause of death was neutropenic sepsis (7/18 deaths). Supportive growth factor was not permitted with cycle 1 of therapy, but management of neutropenia thereafter was at the discretion of the treating physician per guidelines. There were five cardiac-related deaths on the cabazitaxel arm, with none reported in the mitoxantrone-treated subjects. Given the overall benefit, the FDA-approved cabazitaxel for men with mCRPC with disease progression following docetaxel in 2010.

Subsequent use of cabazitaxel, such as in the German Compassionate use Programme, employed closer complete blood count monitoring, with $17.1 \%$ of patients treated with granulocyte colony stimulating factor (G-CSF). ${ }^{7}$ There was $1.8 \%$ incidence of neutropenic fever among 111 patients, with four infection- or hematological-related deaths reported. In the United Kingdom Early Access Programme (UK EAP), 112 docetaxel refractory patients were treated with cabazitaxel $25 \mathrm{mg} / \mathrm{m}^{2} .{ }^{8}$ Primary G-CSF prophylaxis was recommended as per American Society of Clinical Oncology guidelines 
and was administered in $79.5 \%$ of patients initially and, subsequently, a total of $84.8 \%$ of patients received G-CSF. The neutropenic sepsis rate was low $(6.3 \%)$, occurring in patients not treated with prophylactic growth factor, and there were four infection-related deaths. Interestingly, there were no grade $3 / 4$ cardiac events and patients experienced a trend toward pain improvement on self-reported quality of life studies. In a subset analysis of 746 men enrolled in compassionate use and early access programs, safety, as reported based on age, $<70,70-74$, and $>75$ years was reviewed. ${ }^{9}$ In a multivariable analysis, patients aged $>75$ years and those with neutrophil count $<4,000 / \mathrm{mm}^{3}$ at baseline were at the highest risk of neutropenia and complications and prophylactic G-CSF mitigated these risks.

\section{Cabazitaxel dose selection}

The FDA-approved dose of cabazitaxel $\left(25 \mathrm{mg} / \mathrm{m}^{2}\right)$ was based on the TROPIC trial, but questions remained whether a lower dose could still be as efficacious with less toxicity. This led to the FDA-mandated PROSELICA study, a randomized Phase III noninferiority study of cabazitaxel $20 \mathrm{mg} / \mathrm{m}^{2}$ (C20) vs $25 \mathrm{mg} / \mathrm{m}^{2}$ (C25) in 1200 patients with mCRPC previously treated with docetaxel (D). ${ }^{10}$ Of the 10 planned treatments, both groups completed a similar number of median cycles of therapy, 6 (C20) and 7 (C25), with more dose reductions in the $25 \mathrm{mg} / \mathrm{m}^{2} \mathrm{arm}$. There were more grade $3 / 4$ treatmentrelated toxicities and more treatment-related deaths in $\mathrm{C} 25$ compared to $\mathrm{C} 20$. However, a sub group analysis showed that patients treated with prior second-generation antiandrogens (enzalutamide or abiraterone) had a trend toward better outcomes with C25 than C20. Patients treated on the $25 \mathrm{mg} / \mathrm{m}^{2}$ arm had significantly improved PSA response rates $(42.9 \%$ vs $29.5 \%, P<0.001$ ) and improved radiographic response, but there was no difference in PFS. OS was 13.4 months in the C20 arm, which was not inferior to 14.5 months in the C25 cohort (hazard ratio [HR] 20 vs 25, HR=1.024). This met the FDA-mandated prespecified noninferiority endpoint, which maintained $50 \%$ of the OS benefit of the C25 dose in the originally reported TROPIC trial.

Alternative cabaztiaxel dosing regimens that have been explored include a weekly schedule for "unfit" mCRPC patients. Investigators defined unfit as patients with Eastern Cooperative Oncology Group status $>2$, history of dose reduction with docetaxel due to febrile neutropenia, or history of radiation affecting $>25 \%$ of the bone marrow reserve. MTD had previously been established as cabazitaxel $10 \mathrm{mg} / \mathrm{m}^{2}$ given for 4 weeks on a 5 -week cycle. ${ }^{11}$ Sixty-six unfit patients with $\mathrm{mCRPC}$ were evaluated, $87 \%$ of whom had metastatic bone disease. Overall, treatment was well tolerated. The most common grade $3 / 4$ toxicities were asthenia (10.6\%), anemia (6\%), thrombocytopenia (4.5\%), and neutropenia (3\%). There were no occurrences of febrile neutropenia or grade 4 diarrhea. A decline in PSA by $50 \%$ was observed in $32.7 \%$. The median OS with weekly dosing was 14.2 months, making this regimen a reasonable option for patients with an impaired performance status. ${ }^{12}$

\section{Treatment sequencing}

Determining the ideal sequencing of therapy is difficult, as there are no prospective head-to-head trials evaluating all the available treatments for mCRPC. Comparison between individual trials certainly has its own limitations as studies vary in their design, comparator arm, and patient characteristics. As such, clinicians rely heavily on large retrospective analyses to draw conclusions for optimal therapy sequencing post-docetaxel. To date, no clear prospective data exist to support a sequence of subsequent therapy following docetaxel.

\section{Cabazitaxel following second-generation hormone therapy}

Though the sequence and biological rationale for the use of docetaxel followed by cabazitaxel is clear, little is known about resistance mechanisms and optimal sequencing of the other FDA-approved agents for mCRPC. There is a suggestion that resistance to abiraterone may also confer cross resistance to docetaxel, but this is not known for cabazitaxel. ${ }^{13}$ In the aforementioned PROSELICA trial, patients who were previously treated with abiraterone or enzalutamide experienced significantly better rates of PSA response and radiographic responses with cabazitaxel $25 \mathrm{mg} / \mathrm{m}^{2}$ compared to those treated with $20 \mathrm{mg} / \mathrm{m}^{2}$, though no survival differences were noted. These are probably the most robust prospective data on the sequence of cabazitaxel with second-generation androgen inhibitors.

Several retrospective studies have addressed the activity of cabazitaxel following abiraterone. In a multicenter retrospective Israeli study in subjects on abiraterone compassionate use programs following docetaxel, 24 patients received subsequent cabazitaxel for a median of four cycles. ${ }^{14}$ Most patients were treated with growth factor support at the outset of therapy. A PSA response of $>50 \%$ decline from baseline was seen in $31 \%$ of patients, and RECIST response was seen in $13 \%$ of patients, with a median survival of 8.2 months from initiation of cabazitaxel, thus supporting its activity after progression on abiraterone. 
In a single-center retrospective study from the Royal Marsden Hospital, 59 patients who had progressed after docetaxel and who had received cabazitaxel for mCRPC were identified. Thirty-two patients had received prior abiraterone for a median of 7 months, four patients had prior enzalutamide for a median of 1 month, and five patients had received both. ${ }^{15}$ A median of six cabazitaxel cycles were delivered, with an OS of 15.8 months, similar to that reported in the TROPIC study. Lack of response to initial abiraterone and enzalutamide therapy in this retrospective study did not appear to influence the response to cabazitaxel.

In a French and Canadian study, 79 men with mCRPC were treated with a median of six cycles of cabazitaxel $25 \mathrm{mg} / \mathrm{m}^{2}$ post a median of eight cycles of docetaxel and 4.8 months of abiraterone. ${ }^{16}$ Patients experienced an OS of 10.9 months, and PSA decline $>50 \%$ in $35 \%$ of patients.

In a retrospective series from the US Oncology Practice Network, sequences of docetaxel, cabazitaxel, and abiraterone were reviewed. ${ }^{17}$ One hundred thirteen patients received all three agents, of whom 77 patients sequenced docetaxel (D), followed by cabazitaxel (C), followed by abiraterone (A) and 36 patients were treated in the DAC sequence. Patients tolerated more cycles in the DCA compared to the DAC sequence (six vs four cycles, $P<0.001$ ), with improved OS in the DCA sequence as well ( 18.2 vs 11.8 months, $P=0.0023$ ). The authors speculate that the additional cycles of cabazitaxel tolerated may contribute to the clinical benefits of the DCA sequence.

Finally, the impact of previous treatment with secondgeneration androgen inhibition on cabazitaxel efficacy was evaluated in a Phase II Dutch trial of cabazitaxel $25 \mathrm{mg} / \mathrm{m}^{2}$ with prednisone, with or without budesonide to prevent chemotherapy-induced diarrhea. ${ }^{18}$ Forty-four of 114 evaluable patients had received either enzalutamide (3), abiraterone (39), or both (2) following docetaxel, prior to initiating cabazitaxel while the remainder (70) proceeded directly to cabazitaxel after docetaxel failure. Other than slightly lower albumin, there were no baseline differences between those who had and had not received a second-generation therapy. There were no significant differences in rates of PSA response $>50 \%$ to cabazitaxel between the groups treated with and without second-generation antiandrogen agents ( $34 \%$ vs $40 \%, P=0.53$ ), or there was a significant difference in the median OS of 13 vs 14 months.

These retrospective studies help highlight the efficacy of cabazitaxel in mCRPC patients who failed docetaxel and AR-directed therapies, suggesting that the mechanism of resistance for each may be different. Identifying mCRPC patients who may benefit from earlier initiation of cabazitaxel was an integral driving force behind the prospectively designed TAXYNERGY trial. ${ }^{19}$ Investigators looked at overcoming inherent taxane resistance in chemotherapy-naive mCRPC by utilizing an early switch model. Patients previously treated with AR therapies (44.4\%), radiation, or immunotherapy were randomized 2:1 to docetaxel or cabazitaxel and re-evaluated at 12 weeks for PSA decline of $\geq 30 \%$ from baseline. If PSA did not drop sufficiently, they were switched to the other taxanes. Primary endpoint was to improve upon the historical PSA response rate ( $>50 \%$ decline) observed in the TAX327 of $45.4 \%$. In an intention to treat analysis, $55.6 \%$ of patients achieved a PSA decline of $>50 \%$ by the end of the study reaching statistical significance. PSA response rate in patients previously treated with AR therapies was lower (44\%) than AR-naive patients (68\%). Fifteen of 63 patients switched taxane therapy after 12 weeks due to poor PSA response and $46.7 \%$ (7) of these patients achieved a PSA decline of $>50 \%$. Median OS was not reached. Correlation of patient response with circulating tumor cells (CTCs) is described later (refer "Biomarkers of efficacy" section). Further trials to determine benefit of early taxane switching in $\mathrm{MCRPC}$ are warranted with a focus on patients who have failed AR-directed therapies.

\section{Cabazitaxel prior to second-generation hormone therapy}

While cabazitaxel seems to retain activity after exposure to abiraterone or enzalutamide, several studies have evaluated the impact of using it prior to these hormonal agents. In the multicenter retrospective CAST study from the Netherlands, patients with mCRPC following docetaxel were treated with cabazitaxel and abiraterone sequential therapy. ${ }^{20}$ Sixty-three men received cabazitaxel followed by abiraterone (CA), and 69 were treated in the reverse sequence (AC). There was a significant difference in baseline age, with the median age of the CA patients 65.6 years and the mean age of AC patients 69.8 years $(P<0.001)$. Apart from age, there were no other baseline tumor-related differences and both groups received similar number of prior docetaxel cycles. In the CA group, men received a mean of 7.3 cabazitaxel cycles, which was significantly more compared to 4.6 in the AC group $(P<0.001)$. There was no significant difference in OS based on treatment sequence, with median OS 19.1 months for CAtreated patients and 17.0 months for $\mathrm{AC}$-sequenced patients. Hospitalization for febrile neutropenia occurred in 9.5\% CA patients and $14.5 \%$ AC-treated individuals, and there were more deaths within 30 days in the $\mathrm{CA}$ when compared to the $\mathrm{AC}$ sequence, though most were due to disease progression. 
Contrary to the CAST study, the results from a large retrospective Italian study seemed to suggest a slight survival advantage when cabazitaxel (C) was used prior to a secondgeneration novel hormone therapy (NHT) abiraterone and enzalutamide. ${ }^{21}$ Four-hundred seventy-six patients with mCRPC who had received at least two therapies post-docetaxel (D) were grouped by treatment history: D-NHT-C, D-C-NHT, and D-NHT-NHT. The median OS from initiation of the second agent was statistically significant at 12.9 , 14.2 , and 8.8 months, respectively $(P=0.01)$ with the longest OS in the patients treated with cabazitaxel in the second line. Investigators did not report on the median number of cycles of cabazitaxel for each group, but this might be a critical factor that can help explain the OS benefit in the D-C-NHT group.

Similar results were seen with a retrospective review of 574 mCRPC cases from the FLAC international database. ${ }^{22}$ Patients again were separated into groups based on their treatment history: Group 1: D-C-NHT; Group 2: D-NHT-C; and Group 3: D-C. The median number of cycles was 7 for docetaxel and 6 for cabazitaxel. The OS from first docetaxel cycle was 40.1, 37.1, and 30.1 months, respectively. Investigators note that the activity of cabazitaxel did not seem to be influenced by prior NHT use. Although the trend supports improved outcomes with cabazitaxel in the second line, the data are retrospective and the possibility of a patient selection bias toward healthier patients must be recognized. This concern is reinforced by the multivariate analysis that showed patients with a lower PSA, longer ADT response times, and lack of clinical progression at the time of docetaxel initiation overall had a better prognosis.

\section{Rechallenging patients with cabazitaxel}

Data regarding cabazitaxel rechallenge in fit patients with history of good response are limited, but one retrospective study looked at $70 \mathrm{mCRPC}$ patients previously treated with docetaxel (D), cabazitaxel (C), and a second-generation antiandrogen who were retreated with cabazitaxel. ${ }^{23} \mathrm{~A}$ majority of the patients (74\%) had received D-NHT-C, while $24 \%$ received D-C-NHT. The median time from last cabazitaxel dose was 8.6 months. Rechallenge doses of cabazitaxel every 3 weeks were $25 \mathrm{mg} / \mathrm{m}^{2}$ ( $58 \%$ of patients), $20 \mathrm{mg} / \mathrm{m}^{2}$ (27\%), and $16 \mathrm{mg} / \mathrm{m}^{2}(14.3 \%)$. Less than half (47\%) of the patients required growth factor support. The mean PFS was 7.8 months with an OS of 13.4 months from initiation of rechallenge. Grade $3 / 4$ toxicities were $18 \%$, and there were no episodes of febrile neutropenia. As investigators remarked, these promising data may be skewed by patient selection bias, given that all participants were assessed to be fit for chemotherapy despite at least three other lines of therapy.

\section{Cabazitaxel in the first-line treatment of $\mathrm{mCRPC}$}

In 2016, a large-scale trial provided information on cabazitaxel compared to docetaxel for first-line treatment in chemotherapy-naive patients in the FIRSTANA study ${ }^{24}$ This was a three-arm trial comparing two doses of cabazitaxel, 20 and $25 \mathrm{mg} / \mathrm{m}^{2}$, to docetaxel $75 \mathrm{mg} / \mathrm{m}^{2}$ in men with chemotherapy-naive mCRPC. A total of 1168 patients at 159 centers participated, very few of whom were previously treated with second-line hormone therapy. The primary endpoint was OS, and it was hypothesized that cabazitaxel would be superior to docetaxel. The study failed to meet this superiority primary endpoint, as there was no difference in OS between cabazitaxel and docetaxel at either dose, with survival ranging from 24.3 to 25.2 months between the groups, and there was no difference in PFS. The median number of cycles was 9. Dose delays and reductions were highest in the cabazitaxel $25 \mathrm{mg} / \mathrm{m}^{2}$ arm, as were rates of neutropenic fever, infections, diarrhea, and hematuria. Peripheral neuropathy, stomatitis, edema, alopecia, and nail changes were more pronounced in the docetaxel arm compared with the cabazitaxel arm (Table 1). These data continue to support the use of docetaxel as first-line therapy of patients with mCRPC. The discussant of this important and informative Phase III trial, Dr Raghavan, also reviewed the significant cost difference between docetaxel and cabazitaxel, highlighting the value, as well as safety and efficacy of docetaxel in the first line. ${ }^{25}$ More prospective, randomized trials of cabazitaxel in the first line are in progress and will hopefully clarify where it belongs in treatment sequencing of mCRPC.

Table I FIRSTANA (NCTOI308567) side effect profile of docetaxel vs cabazitaxel as first-line treatment in $\mathrm{mCRPC}$

\begin{tabular}{|c|c|c|c|c|}
\hline \multirow[t]{2}{*}{ TEAEs $>5 \%$} & \multicolumn{2}{|c|}{$\begin{array}{l}\text { Docetaxel } \\
75 \mathrm{mg} / \mathrm{m}^{2} \text { (\%) }\end{array}$} & \multicolumn{2}{|c|}{$\begin{array}{l}\text { Cabazitaxel } \\
25 \mathrm{mg} / \mathrm{m}^{2} \text { (\%) }\end{array}$} \\
\hline & All grades & Grade 3/4 & All grades & Grade $3 / 4$ \\
\hline Febrile neutropenia & 8.3 & 8.3 & 12 & 12 \\
\hline Neutropenic infection & 4.9 & 4.1 & 6.1 & 5.9 \\
\hline Diarrhea & 37 & 2.3 & 49.9 & 5.6 \\
\hline Stomatitis & 13.7 & 0.8 & 6.6 & 0.3 \\
\hline Hematuria & 3.6 & 0.3 & 25.1 & 3.6 \\
\hline Peripheral neuropathy & 25.1 & 2.1 & 12.3 & 0 \\
\hline Peripheral edema & 20.4 & 1.6 & 7.7 & 0.3 \\
\hline Alopecia & 39 & 0 & 13 & 0 \\
\hline Nail disorders & 9 & 0.3 & 0.8 & 0 \\
\hline
\end{tabular}

Abbreviations: $\mathrm{mCRPC}$, metastatic castration-resistant prostate cancer; TEAEs, treatment-emergent adverse events. 


\section{Cabazitaxel in combination with a second agent}

Several trials have tried to enhance the antitumor activity of cabazitaxel by adding a second agent with a distinct mechanism of action. Researchers propose that targeting prostate cancer cells in two separate ways will lead to greater cytotoxicity and diminish chances for developing resistance. In the Phase III Affinity trial, investigators randomized $635 \mathrm{mCRPC}$ patients who had failed docetaxel to cabazitaxel \pm curtisen (OGX-011), a novel agent designed to inhibit the production of clusterin, a cytoprotective protein that is upregulated in cancer cells exposed to chemotherapy. ${ }^{26}$ Cabaztiaxel was given once every 3 weeks at $25 \mathrm{mg} / \mathrm{m}^{2}$ and curtisen was given weekly at $640 \mathrm{mg}$ intravenously for a total of 10 cycles. The study unfortunately did not reach its primary endpoint of OS (14.2 vs 13.4 months; $P=0.529$ ).

Cabazitaxel is also being studied with a small-molecule inhibitor tasquinimod in the Phase I CATCH trial. ${ }^{27}$ Tasquinimod limits the activity of myeloid-derived suppressor cells (MDSCs), which accumulate in the tumor microenvironment and inhibit the antitumor activity of $\mathrm{T}$, natural killer, and dendritic cells. The small molecule binds to protein S100A8/A9 and interrupts the positive feedback of further MDSC recruitment. The study established a maximum tolerated dose (MTD) of cabazitaxel $25 \mathrm{mg} / \mathrm{m}^{2}$ and tasquinimod $0.5 \mathrm{mg}$ daily with a lead-in of $0.25 \mathrm{mg}$ daily for 3 weeks. Observed grade $3 / 4$ events were fatigue, febrile neutropenia, and liver dysfunction.

In addition to novel drugs, cabazitaxel is being studied in combination with other agents used in mCRPC. Single-arm, early-phase trials have demonstrated the tolerability and efficacy of cabazitaxel at $25 \mathrm{mg} / \mathrm{m}^{2}$ every 3 weeks with abiraterone $1,000 \mathrm{mg}$ daily. ${ }^{28}$ These include patients previously treated with docetaxel and at least 3 months of abiraterone. Of the 26 patients, $46.2 \%$ achieved a PSA response, which was higher than the historic controls seen with abiraterone alone (29\%) and cabazitaxel alone (39\%). The median PSA-PFS was 6.9 months, and a subset of patients (6) had a sustained PSA response at 6 months. Adverse events observed were similar to those seen with the drugs individually. Additional randomized studies of cabazitaxel and abiraterone are ongoing as are early-phase studies of cabazitaxel with enzalutamide.

Cabazitaxel has also been paired with other chemotherapy agents such as carboplatin with the hypothesis that patients with aggressive variant of prostate cancer (AVPC) may have better outcomes with a taxane-platinum combination. ${ }^{29}$ Previous work by Aparicio et al identified the presence of at least two mutations in p53, Rb1, and/or PTEN in androgen indifferent tumor samples and postulated that their presence would correlated with platinum response. Clinical characteristics of AVPC were defined as histological presence of small cell, visceral, or lytic bone disease at presentation, bulky tumor, or adenopathy, PSA $<10$, despite high disease burden. One hundred sixty men were randomized to cabazitaxel $25 \mathrm{mg} / \mathrm{m}^{2}$ \pm carboplatin at area under the curve 4 every 3 weeks. Median PFS was significantly better in the combination arm at $7.0 \mathrm{vs}$ 4.6 months for cabazitaxel alone $(P=0.004)$. This benefit was greater in those patients with AVPC with median PFS 8 vs 4.5 months $(P=0.0036)$. Molecular profiling of tumor biopsies and ctDNA are in process, but early results suggest that the molecular definition on AVPC better predicts response to platinum-taxane therapy compared to the clinical definition. Investigators are planning a Phase III trial powered for OS.

\section{Biomarkers of efficacy}

In a post hoc analysis of the patients treated with cabazitaxel on the TROPIC study, those who developed grade $>3$ neutropenia had significantly prolonged OS (16.3 vs 14 months, $P=0.035)$, PFS (5.3 vs 2.6 months), and a higher rate of PSA response of $>50 \%$ than those who did not. ${ }^{30}$ There was a trend that the frequency of neutropenia was associated with OS and PFS. The authors also observed the effect of neutrophil-tolymphocyte ratio (NLR) upon outcomes and defined high baseline NLR $>3$ and low $<3$. Analyzed together, those with grade $>3$ neutropenia and low NLR experienced the longest OS of 19.2 months, while those with high NLR and no neutropenia had a 12.9-month OS. In the minority of patients treated with G-CSF after cycle 1 in TROPIC, there did not seem to be an effect upon survival. These authors suggest that a tailored approach to cabazitaxel dosing based on neutropenia may enhance patient outcomes.

With the advent of precision medicine, research has focused on the development of predictive biomarkers to aid in treatment selection. Presence of the AR splice variant 7 (AR-V7) in CTCs from men with mCRPC appears to correlate with treatment resistance to abiraterone and enzalutamide. ${ }^{31}$ Several investigators have sought to determine if the same resistance is true for chemotherapy as well. Thirtyseven men starting either docetaxel or cabazitaxel chemotherapy were prospectively evaluated at a single institution to determine if AR-V7 in CTCs was correlated to clinical outcomes. ${ }^{32}$ There were no significant differences between subjects with and without measurable AR-V7 with respect to response to taxane chemotherapy; however, as expected, AR-V7-positive men responded better to taxanes than to second-generation hormone therapy.

CTCs were also studied in the aforementioned TAXYNERGY trial. Investigators hypothesized that 
taxane-sensitive tumor cells would have a decrease in AR nuclear localization (ARNL) due to disruption of microtubule activity. The ARNL percentage in CTCs was measured on days 1 and 8 of cycle 1 of taxane therapy. A taxane-induced decrease in mean percentage of ARNL significantly correlated with a higher rate of PSA response of $\geq 50 \%$ decline in PSA (72.2\% vs $12.5 \%, P=0.009)$. This evidence supports preclinical data that the anti-tumor activity of taxanes in prostate cancer is related to their disruption of AR trafficking from the cytoplasm to the nucleus and suggests that persistence of ARNL in setting of taxane treatment may be a marker of therapy resistance. ${ }^{19}$ Larger studies are needed to corroborate this potential biomarker of taxane sensitivity.

\section{Patient selection}

The development of treatment predictive biomarkers is a promising field but is still under investigation in prostate cancer. Based on the above studies, cabazitaxel should be reserved off trial for men with mCRPC following docetaxel. Retrospective data suggest that it retains significant activity after progression on second-generation antiandrogens. Proactive management of toxicity, specifically neutropenia, is critical and should especially be considered in patients older than 75 years with baseline absolute neutrophil count $<4,000 / \mathrm{mm}^{3}$. Use of cabazitaxel at lower dose of $20 \mathrm{mg} / \mathrm{m}^{2}$ offers similar OS, but a subset of patients who tolerate treatment with low NLR may have better outcomes. Similarly, a low-dose weekly regimen may be reasonable in patients with Karnofsky Performance Score $<70 \%$. Rechallenging patients with cabazitaxel who previously had a good response is a promising option to help extend survival. With regard to patient preference, prospectively collected data outcomes from the 3,000 men in the Prostate Cancer Registry showed that patients on abiraterone or enzalutamide as second-line treatment after docetaxel reported a higher rate of clinically meaningful improvement in quality of life compared to those treated with cabazitaxel despite similar time to disease progression. ${ }^{33}$ Interestingly, the cabazitaxel group reported lower rates of clinically meaningful deterioration in their quality of life. To date, the time to disease progression is similar among all agents.

Ultimately, more prospective data are needed. Tables 2 and 3 briefly describe the latest trials registered on

Table 2 Recently completed clinical trials of cabazitaxel in mCRPC

\begin{tabular}{|c|c|c|c|}
\hline NCT number & Phase & Description & Results \\
\hline \multicolumn{4}{|c|}{ Single-agent cabazitaxel in mCRPC } \\
\hline NCTOI 254279 & III & $\begin{array}{l}\text { UK EAP to assess quality of life and safety data on } \\
\text { cabazitaxel } 25 \mathrm{mg} / \mathrm{m}^{2} \text { in } \mathrm{mCRPC} \text { previously treated with } \\
\text { docetaxel }^{8}\end{array}$ & $\begin{array}{l}\text { Almost I/3 of patients completed } \geq 10 \text { cycles in the UK } \\
\text { EAP. QOL was stable with trends to improved EQ-5D and } \\
\text { VAS scores. Improved or stable pain was observed in the } \\
\text { majority of patients continuing therapy }\end{array}$ \\
\hline NCT004I 7079 & III & $\begin{array}{l}\text { TROPIC: cabazitaxel vs mitoxantrone in mCRPC } \\
\text { previously treated with docetaxel }{ }^{6}\end{array}$ & $\begin{array}{l}\text { Updated cabazitaxel prolongs OS at } 2 \text { years vs mitoxantrone } \\
\text { and has low rates of peripheral neuropathy. Palliation benefits } \\
\text { of cabazitaxel were comparable to those of mitoxantrone }\end{array}$ \\
\hline NCT0I649635 & IV & $\begin{array}{l}\text { PROSPECTA: to assess effectiveness of prophylactic } \\
\text { treatment of hematological complications (grade } \geq 3 \\
\text { neutropenia) resulting from cabazitaxel treatment in } \\
\text { mCRPC previously treated with docetaxel }\end{array}$ & Not posted \\
\hline NCT02074I37 & IV & $\begin{array}{l}\text { Evaluation of safety of cabazitaxel in patients with } \\
\text { mCRPC previously treated with docetaxel }\end{array}$ & Not posted \\
\hline NCT0244I894 & IV & $\begin{array}{l}\text { PEGAZUS: assess tolerability of cabazitaxel with } \\
\text { primary prophylaxis PEG-G-CSF in mCRPC previously } \\
\text { treated with docetaxel }\end{array}$ & Not posted \\
\hline NCTOI 324583 & I & $\begin{array}{l}\text { Dose-escalation study with cabazitaxel and } \\
\text { prednisolone in patients with hormone refractory } \\
\text { prostate cancer previously treated with docetaxel }\end{array}$ & Not posted \\
\hline \multicolumn{4}{|c|}{ Cabazitaxel dosing in mCRPC } \\
\hline NCTOI 308580 & III & $\begin{array}{l}\text { PROSELICA: cabazitaxel at } 20 \text { vs } 25 \mathrm{mg} / \mathrm{m}^{2} \text { with } \\
\text { prednisone for the treatment of } \mathrm{mCRPC}\end{array}$ & $\begin{array}{l}\text { Cabaztiaxel } 20 \mathrm{mg} / \mathrm{m}^{2} \text { demonstrates noninferiority for } \\
\text { OS compared to Cabaztiaxel } 25 \mathrm{mg} / \mathrm{m}^{2} \text { and an improved } \\
\text { overall safety profile }\end{array}$ \\
\hline NCT0I54I007 & II & $\begin{array}{l}\text { ConCab: assess tolerability of cabazitaxel } 25 \mathrm{mg} / \mathrm{m}^{2} \\
\text { every three weeks vs } 10 \mathrm{mg} / \mathrm{m}^{2} \text { for } 5 \text { consecutive } \\
\text { weeks of a } 6 \text {-week cycle }\end{array}$ & Not posted \\
\hline NCT0I5I8283 & II & Study of weekly cabazitaxel for unfit mCRPC ${ }^{12}$ & $\begin{array}{l}\text { Cabazitaxel } 10 \mathrm{mg} / \mathrm{m}^{2} \text { weekly is tolerable and effective in } \\
\text { unfit patients and results in OS of } 14.2 \text { months }\end{array}$ \\
\hline NCT0I558219 & II & $\begin{array}{l}\text { Safety and efficacy of biweekly dosing of cabazitaxel in } \\
\text { second line treatment of } \mathrm{mCRPC}^{34}\end{array}$ & $\begin{array}{l}\text { Cabazitaxel } 16 \mathrm{mg} / \mathrm{m}^{2} \text { biweekly without G-CSF is tolerable } \\
\text { with } 16 \% \text { neutropenia and } 1.7 \% \text { neutropenic infection }\end{array}$ \\
\hline
\end{tabular}

(Continued) 
Table 2 (Continued)

\begin{tabular}{|c|c|c|c|}
\hline NCT number & Phase & Description & Results \\
\hline \multicolumn{4}{|c|}{ Sequencing of single-agent cabazitaxel in mCRPC } \\
\hline NCTOI7I8353 & II & $\begin{array}{l}\text { TAXYNERGY: explore the benefit of an early switch } \\
\text { from docetaxel to cabazitaxel and vice versa in mCRPC } \\
\text { who do not achieve } \geq 30 \% \text { PSA decline from baseline } \\
\text { by cycle } 4 \text { and correlated with CTCs }{ }^{19}\end{array}$ & $\begin{array}{l}\text { PSA reduction in } 55.6 \% \text { of patients compared to historic } \\
\text { rate of } 45.4 \% \text { in TAX } 327 \text {. Nearly } 90 \% \text { of men with } \\
\text { progressive chemo-naive mCRPC have detectable CTCs } \\
\text { with higher CTC counts associated with adverse prognostic } \\
\text { variables. Lower percent of nuclear AR was associated with } \\
\text { visceral metastases, suggesting that progressive visceral } \\
\text { CRPC may be less AR driven. Decrease in ARNL on day } 8 \\
\text { of taxane treatment correlates with PSA response }\end{array}$ \\
\hline NCT0I576029 & II & SWITCH: compare the continuation of treatment with & Not posted \\
\hline
\end{tabular}

\begin{tabular}{|c|c|c|c|}
\hline \multicolumn{4}{|c|}{ to PSA progression } \\
\hline \multicolumn{4}{|c|}{ Cabazitaxel with AR agent in $\mathrm{MCRPC}$} \\
\hline NCT0I5II536 & I and II & $\begin{array}{l}\text { Determine MTD and efficacy of cabazitaxel with } \\
\text { abiraterone in mCRPC progressed on docetaxel }{ }^{28}\end{array}$ & $\begin{array}{l}\text { Phase I: MTD-cabazitaxel } 25 \mathrm{mg} / \mathrm{m}^{2} \text { every } 2 \mathrm{I} \text { days with } \\
\text { abiraterone I,000 } \mathrm{mg} \text { daily; Phase II: statistically significant } \\
\text { PSA reduction in } 46 \% \text { of patients }\end{array}$ \\
\hline NCT0I845792 & II & $\begin{array}{l}\text { Study of cabazitaxel with or without abiraterone/ } \\
\text { prednisone in mCRPC }\end{array}$ & Not posted \\
\hline \multicolumn{4}{|c|}{ Cabazitaxel with other agents in MCRPC } \\
\hline NCTOI5I3733 & I & $\begin{array}{l}\text { CATCH: determine safety and MTD of tasquinimod } \\
\text { in combination with cabazitaxel in men with } \\
\text { chemorefractory } \mathrm{mCRPC}^{27}\end{array}$ & $\begin{array}{l}\text { MTD established at Cabaztiaxel } 25 \mathrm{mg} / \mathrm{m}^{2} \text { every } 3 \text { weeks } \\
\text { with tasquinimod } 0.5 \mathrm{mg} \text { daily with a } 3 \text {-week lead-in at } \\
0.25 \mathrm{mg} \text { daily }\end{array}$ \\
\hline NCTOI505868 & II & $\begin{array}{l}\text { Study of cabazitaxel with or without carboplatin } \\
\text { (AUC 4) in patients with } \mathrm{mCRPC}^{29}\end{array}$ & $\begin{array}{l}\text { Combination with significantly better PFS overall } \\
(7 \mathrm{vs} 4.6 \mathrm{~m}) \text { and in aggressive subtype ( } 8 \text { vs } 4.5 \mathrm{~m})\end{array}$ \\
\hline NCTOI578655 & III & $\begin{array}{l}\text { AFFINITY: determine if addition of curtisen to cabazitaxel/ } \\
\text { prednisone enhances OS and PFS in } \text { MCRPC }^{26}\end{array}$ & No statistically significant improvement in OS \\
\hline
\end{tabular}
docetaxel vs switching to cabazitaxel regarding the time to PSA progression

Abbreviations: AR, androgen receptor; ARNL, AR nuclear localization; AUC, area under the curve; CRPC, castration-resistant prostate cancer; CTCs, circulating tumor cells; EQ-5D, Descriptive system of health-related quality of life states consisting of five dimensions (mobility, self-care, usual activities, pain/discomfort, anxiety/depression); G-CSF, granulocyte colony stimulating factor; m, months; mCRPC, metastatic castration-resistant prostate cancer; MTD, maximum tolerated dose; OS, overall survival; PEG, polyethylene glycol; PFS, progression-free survival; PSA, prostate specific antigen; QOL, quality of life; UK EAP, United Kingdom Early Access Program; VAS, visual analogue scale.

Table 3 Ongoing clinical trials of cabazitaxel in prostate cancer

\begin{tabular}{|c|c|c|}
\hline NCT number & Phase & Description \\
\hline \multicolumn{3}{|c|}{ Cabazitaxel in high risk or locally advanced disease } \\
\hline NCT01952223 & III & PEACE2: assess effect of neoadjuvant cabazitaxel and pelvic XRT with ADT in high-risk localized prostate cancer \\
\hline NCT0I420250 & 1 & Weekly cabazitaxel with IMRT and ADT in locally advanced prostate cancer \\
\hline NCT0I978873 & III & SenciCab: cabazitaxel + ADT vs ADT alone in metastatic or high-risk disease \\
\hline NCT02543255 & ॥ & ACDC trial: neoadjuvant cabazitaxel and abiraterone with ADT in high-risk prostate cancer \\
\hline \multicolumn{3}{|c|}{ Cabazitaxel sequencing in $\mathrm{mCRPC}$} \\
\hline NCT0I308567 & III & FIRSTANA: cabazitaxel vs docetaxel both with prednisone as first line in patients with $\mathrm{mCRPC}$ \\
\hline NCT02044354 & III & CABA-DOC: patient preference between first-line cabazitaxel vs docetaxel in mCRPC \\
\hline NCT02844582 & II & Cabazitaxel/prednisone as first line therapy in mCRPC \\
\hline NCT02254785 & II & Compare the clinical benefit of cabazitaxel vs abiraterone or enzalutamide in poor prognosis mCRPC patients \\
\hline NCT0248569। & IV & $\begin{array}{l}\text { CARD: compare cabazitaxel vs AR-directed agents in } m \text { CRPC previously treated with docetaxel and who } \\
\text { rapidly failed a prior AR agent }\end{array}$ \\
\hline NCT025I 2458 & II & $\begin{array}{l}\text { CABA-BONE: explore the effect of cabazitaxel on survival pathways and androgen signaling in the tumor } \\
\text { microenvironment (bone marrow) of patients with } \mathrm{mCRPC}\end{array}$ \\
\hline NCT02903I60 & II & $\begin{array}{l}\text { PRINT: determine the clinical benefits of using a rapidly cycling, non-cross-reactive regimen of FDA- } \\
\text { approved prostate cancer therapeutic agents in the management of CRPC }\end{array}$ \\
\hline \multicolumn{3}{|c|}{ Alternate dosing of cabazitaxel in MCRPC } \\
\hline NCT0296/257 & III & $\begin{array}{l}\text { CABASTY: safety of biweekly cabazitaxel at } 16 \mathrm{mg} / \mathrm{m}^{2} \text { vs triweekly cabazitaxel at } 25 \mathrm{mg} / \mathrm{m}^{2} \text { in elderly mCRPC } \\
\text { patients previously treated with docetaxel }\end{array}$ \\
\hline \multicolumn{3}{|c|}{ Cabazitaxel combined with AR agent in MCRPC } \\
\hline NCT02218606 & II & Determine pathologic effects of abiraterone with or without cabazitaxel on mCRPC tissue \\
\hline NCT025227I5 & I and II & Assess safety/tolerability of enzalutamide and cabazitaxel combination in mCRPC \\
\hline NCT03।I 0588 & 1 & $\begin{array}{l}\text { PACE: determine the feasibility and recommended dose of the combination of four drugs (prednisone, } \\
\text { abiraterone, and cabazitaxel and enzalutamide as first-line therapy for mCRPC }\end{array}$ \\
\hline
\end{tabular}


Table 3 (Continued)

\begin{tabular}{|c|c|c|}
\hline NCT number & Phase & Description \\
\hline \multicolumn{3}{|c|}{ Cabazitaxel with other agents in MCRPC } \\
\hline NCTOI594918 & I & CAMP: assess safety and dosing of cabazitaxel with mitoxantrone and prednisone in $\mathrm{mCRPC}$ \\
\hline NCT02703623 & II & $\begin{array}{l}\text { DyanMo: safety and effectiveness of drug combinations in } \mathrm{mCRPC} \text { of apalutamide and abiraterone with } \\
\text { carboplatin and ipilimumab or cabazitaxel }\end{array}$ \\
\hline NCT03043989 & I & Cohorts of docetaxel or cabazitaxel in combination with potent CYP3A4 inhibitor clarithromycin \\
\hline \multicolumn{3}{|c|}{ Biomarker assessment with cabazitaxel } \\
\hline NCT03050866 & II & CABAV7: cabazitaxel in mCRPC patients with AR-V7-positive CTCs \\
\hline NCT03101046 & II & TACTIK: treatment of mCRPC patients according to CTC kinetics \\
\hline
\end{tabular}

Abbreviations: ADT, androgen deprivation therapy; AR, androgen receptor; AR-V7, AR splice variant 7; CRPC, castration-resistant prostate cancer; CTCs, circulating tumor cells; CYP3A4, cytochrome P450 3A4; FDA, US Food and Drug Administration; IMRT, intensity modulated radiation therapy; mCRPC, metastatic castration-resistant prostate cancer; $\mathrm{XRT}$, radiation.

ClinicalTrials.gov that will hopefully provide greater insight on cabazitaxel sequencing as well as alternate dosing patterns or in combination with other therapies.

\section{Conclusion}

Treatment of mCRPC is dynamic and complex. Until the development of predictive treatment biomarkers, clinicians rely on data from prospective clinical trials as well as the conclusions drawn from retrospective analyses. In this review, we examine the evidence for the role of cabazitaxel in mCRPC. The FIRSTANA data continue to support the chemotherapy sequence of docetaxel followed by cabazitaxel. This is also supported by other retrospective studies. However, in looking at past and future studies, it is important to consider that patients sequenced with docetaxel and then cabazitaxel may be fitter, thus confounding the success of DC sequencing. Randomized controlled trials are needed to address this and other variables.

Toxicity from TROPIC and subsequent trials continue to show that treatment-related neutropenia and complications remain significant and can be modified by the use of prophylactic growth factor support, particularly for vulnerable populations of age $>75$ years and baseline absolute neutrophil count $<4,000 / \mathrm{mm}^{3}$. The PROSELICA data suggest that use of cabazitaxel at $20 \mathrm{mg} / \mathrm{m}^{2}$ is perhaps a better value when compared with $25 \mathrm{mg} / \mathrm{m}^{2}$ as it provided a similar survival outcome with less toxicity. This must be weighed against the subgroup analysis showing that patients with prior treatment with abiraterone or enzalutamide had better outcomes on $\mathrm{C} 25$ vs C20. At this time, dosing decisions should continue to be individualized based on the patient's treatment history and performance status.

Future directions of cabazitaxel will be determined by ongoing trials. Its use in combination with AR therapies and other experimental agents may potentially provide better disease control in $\mathrm{mCRPC}$ and help to extend patient survival.

\section{Disclosure}

$\mathrm{JHC}$ is a consultant for Genentech, Clovis Oncology, and Foundation Medicine and reports research support from Sanofi and medical writing services from Genentech. The authors report no other conflicts of interest in this work.

\section{References}

1. American Cancer Society. Cancer Statistics Center; 2016; Available from: https://cancerstatisticscenter.cancer.org/?_ga=1.219304414.651 99597.1475460858\#/. Accessed August 11, 2016.

2. Tannock IF, de Wit R, Berry WR, et al. Docetaxel plus prednisone or mitoxantrone plus prednisone for advanced prostate cancer. $N$ Engl $J$ Med. 2004;351(15):1502-1512.

3. Hurwitz M. Chemotherapy in prostate cancer. Curr Oncol Rep. 2015;17(10):44.

4. Mita AC, Denis LJ, Rowinsky EK, et al. Phase I and pharmacokinetic study of XRP6258 (RPR 116258A), a novel taxane, administered as a 1-hour infusion every 3 weeks in patients with advanced solid tumors. Clin Cancer Res. 2009;15(2):723-730.

5. de Morree E, van Soest R, Aghai A, et al. Understanding taxanes in prostate cancer; importance of intratumoral drug accumulation. Prostate. 2016;76(10):927-936.

6. de Bono JS, Oudard S, Ozguroglu M, et al; TROPIC Investigators. Prednisone plus cabazitaxel or mitoxantrone for metastatic castrationresistant prostate cancer progressing after docetaxel treatment: a randomised open-label trial. Lancet. 2010;376(9747):1147-1154.

7. Heidenreich A, Scholz HJ, Rogenhofer S, et al. Cabazitaxel plus prednisone for metastatic castration-resistant prostate cancer progressing after docetaxel: results from the German compassionate-use programme. Eur Urol. 2013;63(6):977-982.

8. Bahl A, Masson S, Malik Z, et al. Final quality of life and safety data for patients with metastatic castration-resistant prostate cancer treated with cabazitaxel in the UK Early Access Programme (EAP) (NCT01254279). BJU Int. 2015;116(6):880-887.

9. Heidenreich A, Bracarda S, Mason M, et al; European Investigators. Safety of cabazitaxel in senior adults with metastatic castration-resistant prostate cancer: results of the European compassionate-use programme. Eur J Cancer. 2014;50(6):1090-1099.

10. Bono JSD, Hardy-Bessard A-C, Kim C-S, et al. Phase III non-inferiority study of cabazitaxel (C) $20 \mathrm{mg} / \mathrm{m} 2$ (C20) versus $25 \mathrm{mg} / \mathrm{m}^{2}$ (C25) in patients (pts) with metastatic castration-resistant prostate cancer (mCRPC) previously treated with docetaxel (D). J Clin Oncol. 2016;34:5008.

11. Fumoleau $P$, Trigo JM, Isambert N, Sémiond $D$, Gupta $S$, Campone $M$. Phase I dose-finding study of cabazitaxel administered weekly in patients with advanced solid tumours. BMC Cancer. 2013;13(1):460.

12. Calvo OF, Parra EF, Perez-Valderrama B, et al. Weekly cabazitaxel in "unfit" metastatic castration-resistant prostate cancer patients (mCRPC) progressing after docetaxel (D) treatment: preliminary results of CABASEMSOGUG phase II trial. J Clin Oncol. 2015;33(suppl 7):Abstract167. 
13. van Soest RJ, de Morree ES, Kweldam CF, et al. Targeting the androgen receptor confers in vivo cross-resistance between enzalutamide and docetaxel, but not cabazitaxel, in castration-resistant prostate cancer. Eur Urol. 2015;67(6):981-985.

14. Sella A, Sella T, Peer A, et al. Activity of cabazitaxel after docetaxel and abiraterone acetate therapy in patients with castration-resistant prostate cancer. Clin Genitourin Cancer. 2014;12(6):428-432.

15. Pezaro CJ, Omlin AG, Altavilla A, et al. Activity of cabazitaxel in castration-resistant prostate cancer progressing after docetaxel and next-generation endocrine agents. Eur Urol. 2014;66(3): 459-465.

16. Al Nakouzi N, Le Moulec S, Albiges L, et al. Cabazitaxel remains active in patients progressing after docetaxel followed by novel androgen receptor pathway targeted therapies. Eur Urol. 2015;68(2): 228-235.

17. Sonpavde G, Bhor M, Hennessy D, et al. Sequencing of cabazitaxel and abiraterone acetate after docetaxel in metastatic castration-resistant prostate cancer: treatment patterns and clinical outcomes in multicenter community-based US oncology practices. Clin Genitourin Cancer. 2015;13(4):309-318.

18. van Soest RJ, Nieuweboer AJM, de Morrée ES, et al; Dutch UroOncology Study group (DUOS). The influence of prior novel androgen receptor targeted therapy on the efficacy of cabazitaxel in men with metastatic castration-resistant prostate cancer. Eur J Cancer. 2015; 51(17):2562-2569.

19. Antonarakis ES, Tagawa ST, Galletti G, et al; TAXYNERGY Investigators. Randomized, noncomparative, phase II trial of early switch from docetaxel to cabazitaxel or vice versa, with integrated biomarker analysis, in men with chemotherapy-naïve, metastatic, castrationresistant prostate cancer. J Clin Oncol. Epub 2017 Jun 20.

20. Wissing MD, Coenen JL, van den Berg P, et al. CAST: a retrospective analysis of cabazitaxel and abiraterone acetate sequential treatment in patients with metastatic castrate-resistant prostate cancer previously treated with docetaxel. Int J Cancer. 2015;136(6):E760-E772.

21. Caffo O, Bria E, Giorgi UD, et al. Outcomes of metastatic castrationresistant prostate cancer (mCRPC) patients (pts) treated with different new agents (NAs) sequence in post-docetaxel (DOC) setting: final analysis from a multicenter Italian study. J Clin Oncol. 2017;35: Abstract5030.

22. Delanoy N, Angelergues A, Efstathiou E, et al. Sequencing in metastatic castration-resistant prostate cancer (mCRPC): updated results of the FLAC International Database. J Clin Oncol. 2017;35:Abstract267.

23. Thibault C, Eymard J-C, Hardy-Bessard A-C, et al. Efficacy of cabazitaxel (CABA) rechallenge in heavily-treated patients with metastatic castration-resistant prostate cancer (mCRPC). J Clin Oncol. 2017;35:Abstract5033.
24. Oudard S, Fizazi K, Sengeløv L, et al. Cabazitaxel versus docetaxel as first-line therapy for patients with metastatic castration-resistant prostate cancer: a randomized Phase III trial-FIRSTANA. J Clin Oncol. In press 2017.

25. Levitan D. Cabazitaxel No Better Than Docetaxel in Metastatic CRPC; 2016. Available at: http://www.cancernetwork.com/asco-prostatecancer/cabazitaxel-no-better-docetaxel-metastatic-crpc. Accessed October 1, 2016.

26. Fiazi K, Hotte SJ, Saad F, et al. Final overall survival (OS) from the AFFINITY phase 3 trial of custirsen and cabazitaxel/prednisone in men with previously treated metastatic castrate resistant prostate cancer. Ann Oncol. 2016;27(6):1-36.

27. Armstrong AJ, Humeniuk MS, Healy P, et al. Phase Ib trial of cabazitaxel and tasquinimod in men with heavily pretreated metastatic castration resistant prostate cancer (mCRPC): the CATCH trial. Prostate. 2017;77(4):385-395.

28. Massard C, Mateo J, Loriot Y, et al. Phase I/II trial of cabazitaxel plus abiraterone in patients with metastatic castration-resistant prostate cancer (mCRPC) progressing after docetaxel and abiraterone. Ann Oncol. 2017;28(1):90-95.

29. Aparicio A, Xiao L, Tapia ELN, et al. The aggressive variant prostate carcinoma (AVPC) molecular signature (-MS) and platinum-sensitivity in castration resistant prostate cancer (CRPC). J Clin Oncol. 2017;36: Abstract5013.

30. Meisel A, von Felten S, Vogt DR, et al. Severe neutropenia during cabazitaxel treatment is associated with survival benefit in men with metastatic castration-resistant prostate cancer (mCRPC): a post-hoc analysis of the TROPIC phase III trial. Eur J Cancer. 2016;56:93-100.

31. Antonarakis ES, Lu C, Wang $\mathrm{H}$, et al. AR-V7 and resistance to enzalutamide and abiraterone in prostate cancer. $N$ Engl J Med. 2014; 371(11):1028-1038.

32. Antonarakis ES, Lu C, Luber B, et al. Androgen receptor splice variant 7 and efficacy of taxane chemotherapy in patients with metastatic castration-resistant prostate cancer. JAMA Oncol. 2015;1(5):582-591.

33. Chowdhury S, Birtle AJ, Bjartell A, et al. Real-world outcomes in second-line treatment of metastatic castration-resistant prostate cancer (mCRPC): The Prostate Cancer Registry. J Clin Oncol. 2017;35: Abstract5038.

34. Kellokumpu-Lehtinen P-L, Marttila T, Jekunen AP, et al. Biweekly cabazitaxel as a safe treatment option for metastatic castration resistant prostate cancer (mCRPC) patients post-docetaxel: Final analysis of Prosty II trial. J Clin Oncol. 2016;34(15_suppl):e16523-e16523.
OncoTargets and Therapy

\section{Publish your work in this journal}

OncoTargets and Therapy is an international, peer-reviewed, open access journal focusing on the pathological basis of all cancers, potential targets for therapy and treatment protocols employed to improve the management of cancer patients. The journal also focuses on the impact of management programs and new therapeutic agents and protocols on

\section{Dovepress}

patient perspectives such as quality of life, adherence and satisfaction The manuscript management system is completely online and includes a very quick and fair peer-review system, which is all easy to use. Visit http://www.dovepress.com/testimonials.php to read real quotes from published authors. 